\title{
Evaluation of vaginal antifungal formulations in vivo
}

\author{
R. J. MCRIPLEY \\ Ph.D. \\ R. A. SCHWIND \\ B.S.
}

\author{
P. J. ERHARD \\ B.S.
}

R. R. WHITNEY

B.A.

The Squibb Institute for Medical Research, Princeton, New Jersey, U.S.A.

\begin{abstract}
Summary
Relatively simple and rapid procedures have been developed for evaluating the local efficacy of vaginal antifungal agents in vivo in a vaginal candidiasis model in ovariectomized rats. The results of this investigation indicate that the model and methods described are quite suitable for screening potential antifungal substances and for assessing the chemotherapeutic effectiveness of new antifungal agents and formulations before carrying out clinical studies.
\end{abstract}

\section{Introduction}

Vaginal candidiasis is a common and chronic ailment in women of child-bearing age. The incidence of this vaginal infection has increased considerably in recent years with the more frequent use of antibiotics and oral contraceptives. Pregnancy and diabetes are thought to be specific predisposing factors. While a few good antifungal agents, formulated as vaginal products, are available for treating vaginal candidiasis, there is still a need for safer and more efficacious vaginal preparations. Consequently, efforts to discover new and better antifungal agents and to prepare more effective vaginal formulations have continued. The authors recently developed relatively simple and rapid test procedures for evaluating the efficacy of new vaginal antifungal agents and formulations using an experimental vaginal candidiasis in ovariectomized rats.

\section{Materials and methods \\ Animals}

Female, ovariectomized Charles River CD rats (Sprague-Dawley derived) weighing $190 \pm 10 \mathrm{~g}$ were used in all studies. Pairs of rats were caged on wire in temperature- and humidity-controlled rooms and permitted food and water ad libitum.

\section{Test organism}

Candida albicans SC 5314, a clinical isolate maintained in the departmental culture collection, was employed to establish the experimental infection. Inocula for infection were prepared by harvesting the growth from Sabouraud's dextrose agar slants of $C$. albicans obtained after $24-48 \mathrm{hr}$ incubation at $37^{\circ} \mathrm{C}$ and making suspensions containing $10^{8}$ colonyforming units (CFU)/ml.

\section{Establishment of the experimental infection}

Rats were pretreated with $0.5 \mathrm{mg}$ of progesterone and $0.5 \mathrm{mg}$ of oestradiol valerate administered sub cutaneously to induce oestrus. Confirmation of oestrus was made by microscopic examination of vaginal smears. Beginning 2 days after hormona treatment, rats were inoculated intravaginally on 2 successive days with $10^{7} \mathrm{CFU}$ of $C$. albicans cons tained in a $0.1 \mathrm{ml}$ inoculum. The presence of vaginat candidiasis in inoculated rats was confirmed microscopically and culturally.

Quantitation of C. albicans in the experimental infection

At various intervals after infection, an estimate of the number of candidal CFU in the vagina was determined by culture of saline washings obtained by vaginal lavage. Each wash suspension was appropriately diluted and then plated on Mycosel agar (BBL) using conventional plating techniques. Plates were incubated for $48 \mathrm{hr}$ at $37^{\circ} \mathrm{C}$, after which colony counts were performed using an electronic colony counter. For a few experiments, groups of rats were sacrificed at various intervals after infection, and the genital tract of each rat removed in toto. Each organ was dissected into 3 parts; the vagina, the cervix (including the body of the uterus), and the uterine horns. The various tissues were homogenized individually and appropriate dilutions of each homogenate were plated on Mycosel agar. For histological studies, the intact genital tract or the vagina alone was placed in $10 \%$ formalin, embedded in paraffin, sectioned and stained with haematoxylin and eosin or periodic acid-Schiff stain. 


\section{Chemotherapeutic studies}

The following commercial antifungal products were studied: AVC cream, $15 \%$ sulphanilamide and $0.2 \%$ aminacrine (Merrel-National Laboratories); Betadine vaginal gel, $10 \%$ povidone-iodine (Purdue Frederick Co.); Candeptin vaginal ointment, 0.6 $\mathrm{mg} / \mathrm{g}$ candicidin (Schmid Laboratories); GyneLotrimin vaginal tablets, $100 \mathrm{mg}$ clotrimazole/ tablet (Delbay Pharmaceuticals); Monistat 7 vaginal cream, $2 \%$ miconazole nitrate (Ortho Pharmaceutical Corp.); mycostatin cream, 100000 u./g nystatin (E. R. Squibb and Sons); Sporostacin cream, 1\% chlordantoin and $0.05 \%$ benzalkonium chloride (Ortho Pharmaceutical Corp.); and Tricandil candelette vaginali, $25000 \mathrm{u}$. partricin methyl ester/ ovule (Società Prodotti Antibiotici).

Three days after the final intravaginal inoculation of C. albicans, rats were randomly divided into groups of 10-20 animals and treatment was begun. Approximately $0.1 \mathrm{~g}$ of a cream, ointment or gel formulation was placed high in the vaginal vault by means of a ball-tipped needle and syringe. Formulations were administered twice daily for 3 days. Solid dosage forms (tablets and ovules) were inserted intravaginally in reformulated rat-size portions once daily for 3 days. Forty-two hours after the final treatment vaginal washings were obtained from all rats, and appropriate dilutions were cultured as described above. The response of the infection to chemotherapy was determined by comparing the mean candidal count for each treatment group to that obtained from a placebo-treated control group.

\section{Detection of residual drug activity}

Vaginal washings, obtained from rats $42 \mathrm{hr}$ after the termination of therapy were passed through $0.45-\mu \mathrm{m}$ membrane filters before being tested for drug activity. Aliquots of washings were incubated at $37^{\circ} \mathrm{C}$ in the presence of known concentrations of C. albicans $\left(10^{3}\right.$ to $\left.10^{5} \mathrm{CFU} / \mathrm{ml}\right)$. At various intervals during the incubation period, samples were removed from each mixture, diluted if necessary, and plated on Sabouraud's dextrose agar. The mean concentration of candidal CFU determined for each group of test mixtures was compared to that obtained with control mixtures containing washings from a placebo-treated or untreated group of rats. Filtrates of washings from treated groups were sometimes plated directly on agar plates along with an inoculum containing a known concentration of $C$. albicans to determine if there was drug carry-over.

\section{Results}

\section{Experimental infection}

The vaginal infection of ovariectomized rats with C. albicans SC 5314 was studied macroscopically, microscopically and culturally. Grossly, no signs of infection were evident throughout the course of several long-term experiments. Vaginal discharges or plaques on the vaginal mucosa, commonly found in human candidal vaginitis, were not observed. By microscopic examination $24 \mathrm{hr}$ after infection the vaginal mucosa was found to exhibit the classical signs of oestrus; marked hyperkeratosis and pronounced exfoliation of the stratum corneum. Blastospores of $C$. albicans were observed in the superficial layers of the vaginal mucosa and lying free in the vaginal cavity. A slight inflammatory response was noted in the subcutaneous tissues. By $72 \mathrm{hr}$ after infection the concentration of blastospores appeared to have diminished but a few mycelial elements were observed in the stratum corneum.

The magnitude and extent of vaginal colonization by $C$. albicans was followed for several weeks using culture of vaginal washings to quantitate candidal CFU (Fig. 1). The peak concentration of C. albicans

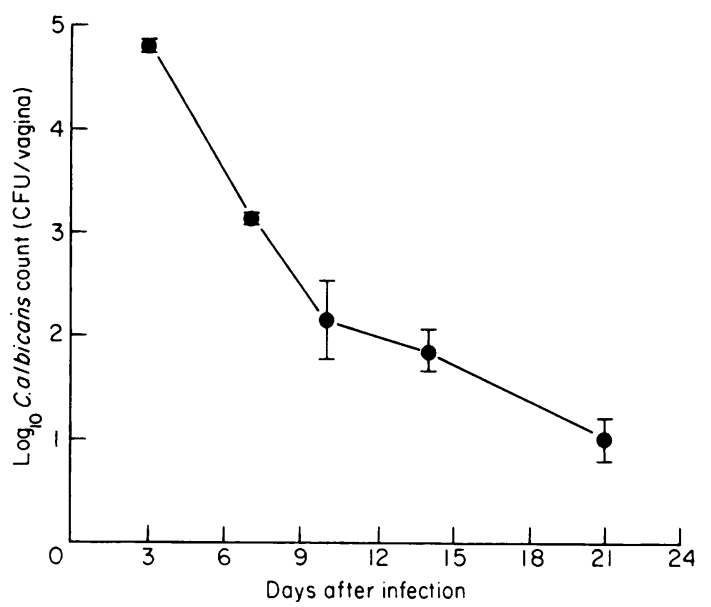

FIG. 1. Time course of experimental vaginal candidiasis (CFU, colony-forming units).

was found $24 \mathrm{hr}$ after infection; however, during the remainder of the first week, the candidal count fell rather precipitously; thereafter, the count declined more gradually. By day 30, C. albicans was seldom isolated from vaginal washings. Small numbers of candidal cells were isolated from extravaginal sites in the genital tract at various intervals after infection.

\section{Sampling methods}

Before employing the acute vaginal candidiasis model for chemotherapeutic studies, various methods of quantitating vaginal C. albicans were investigated. The use of swabs for sampling the vaginal candidal count was found to be unsatisfactory. Homogenization and culture appeared to be the method of 
choice for determining the vaginal candidal count. However, this method required much effort and was quite time consuming. Thus, vaginal lavage and culture, which has been widely used for clinical studies, was investigated as a potential method for sampling. The accuracy of the vaginal lavage and culture method of sampling was evaluated by comparing results obtained by that method to those obtained using vaginal homogenization and culture (Table 1). As expected, higher candidal counts were consistently obtained from cultures of vaginal homogenates. However, neither individual counts from vaginal homogenates nor the mean count of a group were seldom more than one $\log _{10}$ higher than comparable counts obtained by cultures of vaginal washings. Thus, it appeared that sampling by vaginal lavage, while not as accurate as the homogenization technique, nevertheless demonstrated somewhat similar variation and a sufficiently high recovery rate to warrant its routine use.

\section{Response to chemotherapy}

In order to determine if the experimental infection would respond to chemotherapy, various commercial antifungal formulations were evaluated. The results of a typical experiment are shown in Table 2. As can be seen, the infection did respond to chemotherapy, exhibiting different responses to different products. It should be noted that the primary evaluation of efficacy is based on the mean candidal count and the resulting per cent. reduction relative to the placebo control. These results and others, not shown here, indicated that the model infection could be used for chemotherapeutic studies. However, in order fully to characterize the model and its response to chemotherapy, dose-response studies with clotrimazole, nystatin, and miconazole were conducted. Good dose-response curves were obtained with the 3 antifungal agents (Fig. 2).

\section{Residual drug activity}

Experiments were carried out to ensure that any response of the experimental infection to chemo- therapy was due to actual drug efficacy resulting from treatment and not to residual drug or drugcarry-over that would reduce candidal counts during the plating procedure. Cell-free filtrates of washings from rats treated with each drug evaluated were tested for residual drug activity and/or drug carryover to the culture medium. In no instance was there any suggestion that these possibilities might be occurring in the evaluation of various chemotherapeutic agents.

\section{Discussion}

In order to identify and evaluate substances that may be effective as vaginal antifungal agents in humans, experimental infections in laboratory animals have been developed. Scholer (1960) reported on the establishment and practical applications of a vaginal candidiasis model in ovariectomized rats. A similar model in mice was described by Taschdjian, Reiss and Kozinn (1960), but it remained for Wildfeuer (1974) to adapt the model to chemotherapeutic studies. Because several investi gators have demonstrated the utility of the rat mode $\bar{B}$

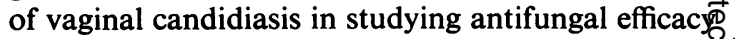
(Mizuba et al., 1974; Polak, 1978), the presen $\$$ authors selected the model for further characteriza tion and subsequent determination as to its potentiat as a screening tool for potential antifungal agents.

The results obtained with several known anti fungal agents, when evaluated in the model infection using test procedures such as the 3-day treatment $t^{+}$ schedule, culture of vaginal washings, and doseresponse studies, indicate that relatively simple, rapid, and effective means of screening have been developed. In addition, it was found that statistical analyses of data generated with the test procedures and the model and the incorporation of the findings into the experimental design can lead to a more significant evaluation of potential antifungal agents. For example, data from dose-response studies can be utilized to indicate sample size requirements or to

TABLE 1. Comparison of 2 methods of sampling vaginal candidal counts

\begin{tabular}{ccccc}
\hline \multirow{2}{*}{$\begin{array}{c}\text { Experiment } \\
\text { no. }\end{array}$} & $\begin{array}{c}\text { Post-infection } \\
\text { day }\end{array}$ & $\begin{array}{c}\text { No. of rats } \\
\text { per group }\end{array}$ & Vaginal lavage & Vaginal homogenate \\
\cline { 3 - 5 } & 3 & 10 & $6.85 \pm 3.17 \times 10^{4}$ & $3.00 \pm 2.01 \times 10^{5}$ \\
1 & 7 & 10 & $3.02 \pm 1.33 \times 10^{4}$ & $1.15 \pm 0.36 \times 10^{5}$ \\
& 14 & 10 & $8 \cdot 52 \pm 3.37 \times 10^{3}$ & $1.89 \pm 0.75 \times 10^{4}$ \\
& 21 & 12 & $1.36 \pm 0.68 \times 10^{3}$ & $3.58 \pm 1.32 \times 10^{4}$ \\
2 & 3 & 6 & $1.45 \pm 0.32 \times 10^{5}$ & $6.97 \pm 1.60 \times 10^{5}$ \\
& 7 & 6 & $3.26 \pm 0.96 \times 10^{4}$ & $1.82 \pm 0.72 \times 10^{5}$ \\
& 14 & 6 & $1.68 \pm 1.04 \times 10^{4}$ & $7.05 \pm 2.65 \times 10^{4}$ \\
& 21 & 6 & $3.22 \pm 1.28 \times 10^{3}$ & $2.86 \pm 1.23 \times 10^{4}$ \\
\hline
\end{tabular}


TABLE 2. Responsiveness of experimental vaginal candidiasis to local chemotherapy with various marketed antifungal products

\begin{tabular}{|c|c|c|}
\hline Test formulation (active ingredient) & $\begin{array}{l}\text { Treatment } \\
\text { regimen }\end{array}$ & $\begin{array}{l}\text { Per cent. reduction } \\
\text { in candidal count* }\end{array}$ \\
\hline $\begin{array}{l}\text { AVC cream } \\
\text { (sulphanilamide, } 15 \% \text {; and } \\
\text { aminacrine } \mathrm{HCl}, 0 \cdot 2 \% \text { ) }\end{array}$ & $0 \cdot 1 \mathrm{~g} \ddagger$ & $97 \cdot 5$ \\
\hline $\begin{array}{l}\text { Betadine vaginal gel } \\
\text { (povidone-iodine, } 10 \% \text { ) }\end{array}$ & $0.1 \mathrm{~g} \ddagger$ & $43 \cdot 3$ \\
\hline $\begin{array}{l}\text { Candeptin vaginal ointment } \\
\text { (candicidin, } 0.6 \mathrm{mg} / \mathrm{g} \text { ) }\end{array}$ & $0 \cdot 1 \mathrm{~g} \ddagger$ & $87 \cdot 0$ \\
\hline $\begin{array}{l}\text { Gyne-Lotrimin vaginal tablets } \\
\text { (clotrimazole, } 100 \mathrm{mg} / \text { tablet) }\end{array}$ & $\begin{array}{l}50 \mathrm{mg} \text { tablet } \\
(4 \mathrm{mg} \text { clotrim. }) \S\end{array}$ & 98.9 \\
\hline $\begin{array}{l}\text { Monistat } 7 \text { vaginal cream } \\
\left(\text { miconazole } \mathrm{NO}_{3}, 2 \%\right)\end{array}$ & $0 \cdot 1 \mathrm{~g} \ddagger$ & $97 \cdot 9$ \\
\hline $\begin{array}{l}\text { Mycostatin cream } \\
\text { (nystatin, 100,000 units/g) }\end{array}$ & $0 \cdot 1 \mathrm{~g} f$ & $95 \cdot 6$ \\
\hline $\begin{array}{l}\text { Sporostacin cream } \\
\text { (chlordantoin, } 1 \% \text {, and benzalkonium } \mathrm{Cl}, 0.05 \% \text { ) }\end{array}$ & $0 \cdot 1 \mathrm{~g} \ddagger$ & $51 \cdot 3$ \\
\hline $\begin{array}{l}\text { Tricandil vaginal candelettes } \\
\text { (partricin methyl ester (PME), } 25000 \text { u./ovule) }\end{array}$ & $\begin{array}{l}56 \mathrm{mg} \text { ovule } \\
(778 \mathrm{u} . \mathrm{PME}) \S\end{array}$ & $86 \cdot 4$ \\
\hline Placebo cream & $0 \cdot 1 \mathrm{~g} \ddagger$ & - \\
\hline
\end{tabular}

* Relative to the mean candidal count in the placebo-treated group.

$\ddagger=$ twice daily $\times 3$.

$\S=$ every day $\times 3$.

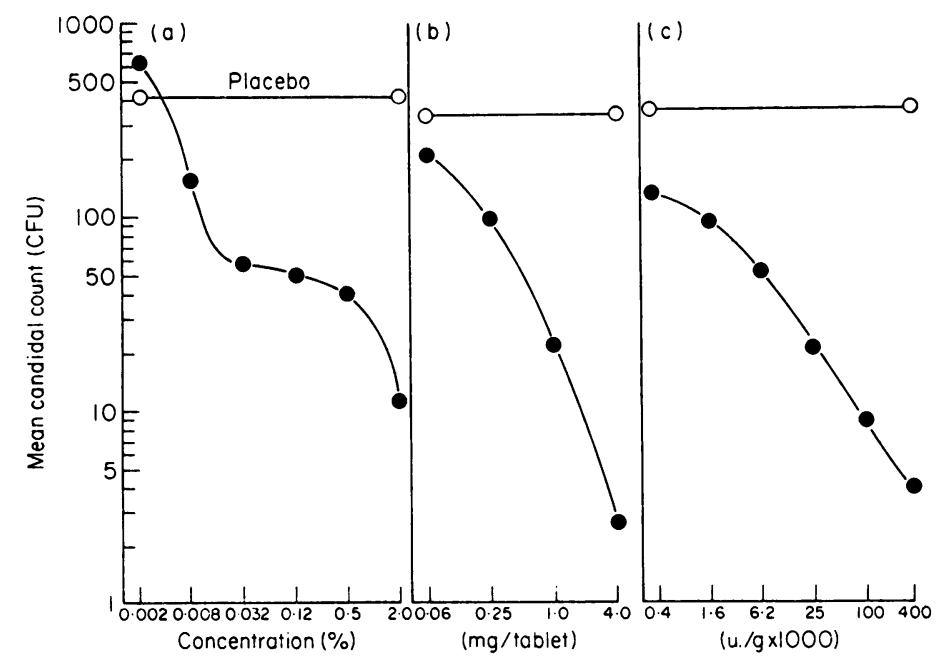

Fig. 2. Dose-response studies with known antifungal agents in the vaginal candidiasis model (CFU, colony-forming units). (a) Miconazole (cream), (b) clotrimazole (tablets) and (c) nystatin (cream); - antifungal agent, $\bigcirc-\bigcirc$ placebo. 
determine by means of a parallel line assay the potency of a test drug relative to a reference drug.

Scholer (1960) reported that the vaginal candidiasis model in ovariectomized rats responded to chemotherapy with antifungal agents when they were administered systemically or locally. In the present study it was found that the model infection responded to a wide variety of antifungal agents applied locally in cream, ointment or gel formulations. It was also demonstrated that the model infection would respond to chemotherapy with both solid and liquid drug formulations (Mizuba et al., 1974). Consequently, one aspect of the rat model that enhances its effectiveness is its capacity to respond to chemotherapy with various types of antifungal agents administered by different routes and in diverse dosage forms.

As with most model infections, there are certain differences between the experimental infection and the natural infection. For example, the experimental infection is rather acute and self-limiting when compared to the chronic, persistent ailment found in women; the experimental infection does not exhibit vulval involvement, vaginal discharges, or mural plaques as is often observed in women; the vaginal $\mathrm{pH}$ in the experimental infection is less acidic than in the natural infection; and the normal flora and vaginal mucosa are somewhat different in the 2 hosts. On the other hand, the experimental infection somewhat resembles the natural infection in that candidal elements are localized superficially in the vagina, both yeast and mycelial forms of $C$. albicans are found, and a microenvironment similar to that found in humans (mixed bacterial flora, vaginal secretions, and $\mathrm{pH}$, etc.) is present. The rat model provides some of the same variables encountered clinically; individual variations in vaginal size and shape, ecosystems, responsiveness to chemotherapy, and retention of drugs.

While differences between the experimental and natural infection do exist, the model does meet various criteria for use as a successful screening tool: (i) the infection is easily produced and quantitated, (ii) the infection is responsive to clinically effective drugs, (iii) the infection can demonstrate doseresponse relationships, and indicate relative potency, and (iv) results from chemotherapeutic studies in the model appear to have some predictive value for clinical studies. Thus, using the test procedures described earlier, the vaginal candidiasis model would appear to have a definite role as a screening tool in the preclinical evaluation of potential antifungal agents.

\section{Acknowledgments}

We thank Sandra Kesckes, William Morgans, and Rocco George for technical assistance.

References

Mizuba, S., Muir, R.D., Johnson, F., Lee, K. \& Ziecina, S

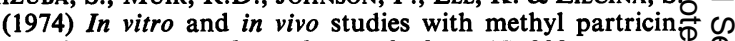
Developments in Industrial Microbiology, 15, 338.

Polak, A. (1978) Synergism of polyene antibiotics with 5 fluorocytosine. Chemotherapy, 24, 2.

SCHOLER, H.J. (1960) Experimentelle vaginal-Candidiasis der Ratte. Pathologia et microbiologia, 23, 62.

Taschdjian, C.L., Reiss, F. \& KozinN, P.J. (1960) Experio mental vaginal candidiasis in mice; its implications fo superficial candidiasis in humans. Journal of Investigative Dermatology, 34, 89.

WiLdFEUER, A. (1974) Die Chemotherapie der vaginalen Trichomoniasis und Candidiasis der Maus. ArzneimittelForschung, 24, 937. 